\title{
Estimation of Friction Force in Minimally Invasive Surgery with Tactile Sensors
}

\author{
${ }^{1}$ Mithat Can Özin, ${ }^{2}$ Bilsay Sümer and ${ }^{* 1}$ İlker Murat Koç \\ ${ }^{* 1}$ Dept. of Mech. Eng., Istanbul Technical University, Gümüşsuyu, 34437, Beyoğlu, İstanbul, Turkey \\ ${ }^{2}$ Dept. of Mech. Eng., Hacettepe University, Beytepe, 06800, Ankara, Turkey
}

\begin{abstract}
:
This study investigates coefficient of friction of flat-tipped laparoscopic surgery tool (bowel grasper) in an ex vivo experiment in order to identify the approximate coefficient of friction between laparoscopic tool and the organic sample. The estimation of friction force is essential for ensuring safe grasping. The friction tribometer and the laparoscopic grasper setup were the two experimental setups to conclude the friction of the tool in the absence tactile sensor. The measurement of the pinch force and estimation of the friction force from the measured pinch force and the coefficient of friction is suitable for novice surgeon training.
\end{abstract}

Key words: Laparoscopic grasper, Coefficient of Friction, Friction of Tissue

\section{Introduction}

Novice surgeons tends to apply more grasping force than experienced surgeons do during minimal invasive surgery owing to practice of special surgical tools so called laparoscopic tools. Hence, the special surgical tools weakens tactile senses of the novice surgeons. Improvement of the surgeon's tactile senses while using special surgical tools is only possible by training and practice. Integration of the novel sensors such as thin film tactile sensors to the surgical tools and creating training platforms equipped with these tools can benefit novice surgeons to enhance their skills and senses [1-3]. Thin film tactile sensors can be used for measuring contact force and pressure in real-time for minimally invasive surgery [4-8]. Integration of a thin layer to tool alters contact characteristics in despite of its advantages.

Friction force measurement is crucial to avoid slippage of tissue from the tool and coefficient of friction is an essential parameter to examine the friction characteristic of the contacting surfaces [9]. Changing velocity and normal force and surface geometry have effect on coefficient of friction between the tool and the tissues which exhibits hyper viscoelastic characteristics [10-14]. In this work, coefficient of friction of flat-tipped laparoscopic surgery tool (bowel grasper) in an ex vivo experiment are studied in order to identify the approximate coefficient of friction between laparoscopic tool and the organic sample. This study is organized as follows: Section 2 introduces the materials and methods including laparoscopic grasping and the experimental setups. In Section 3 , the results of the experiments are given. The discussion of the experimental results are presented in Section 4. The conclusions are given in Section 5.

*Corresponding author: Address: Dept. of Mech. Eng., Istanbul Technical University, Gümüşsuyu, 34437, Beyoğlu, İstanbul, TURKEY, E-mail address: kocilk@itu.edu.tr, Phone: +902122931300/Int:2685 


\section{Materials and Method}

\subsection{Forces in Laparoscopic Grasping}

Main forces to calculate the coefficient of friction during laparoscopic grasping are pinch and pull forces as shown in Fig.1. The pinch force is normal force applied from tool tip and the friction force is orthogonal to the pinch force. Additionally, the combination of pinch and friction force creates pull force, which changes with the closing angle of the end-effector $(\theta)$. Pinch force can be measured by a tactile sensor at the tool tip [15]. A supplementary sensor is necessary to measure the pull force. Coefficient of friction can be calculated if pinch force, pull force and angular displacement of the jaws are measured.

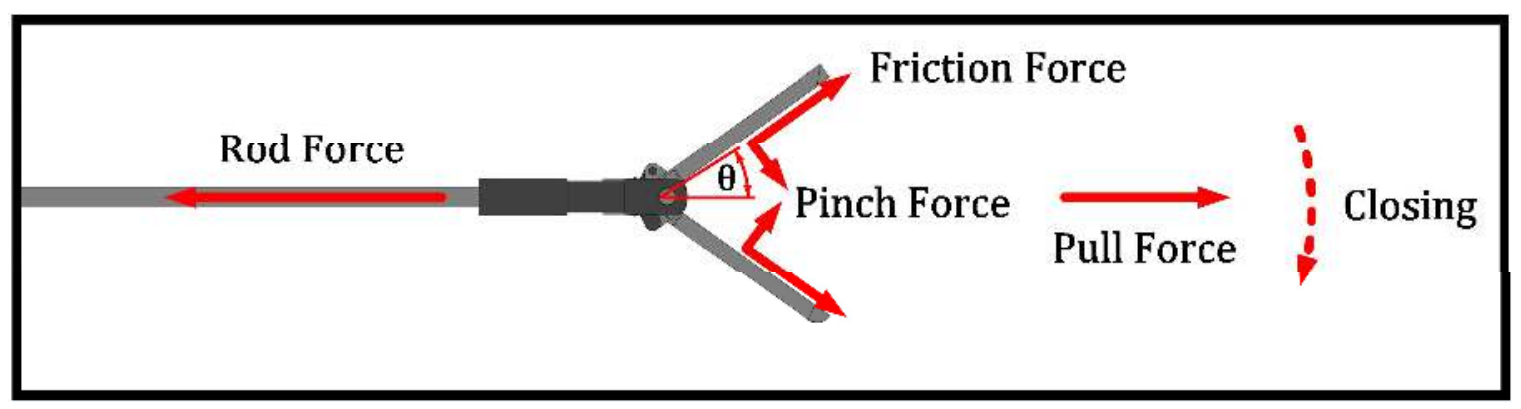

Figure 1. Forces during grasping for closure of jaws.

\subsection{Experimental Setups}

In this subsection, details of two experimental setups, which are friction tribometer and laparoscopic grasper friction setup, are given in order to determine the coefficient of friction of the flat-tipped laparoscopic grasper.

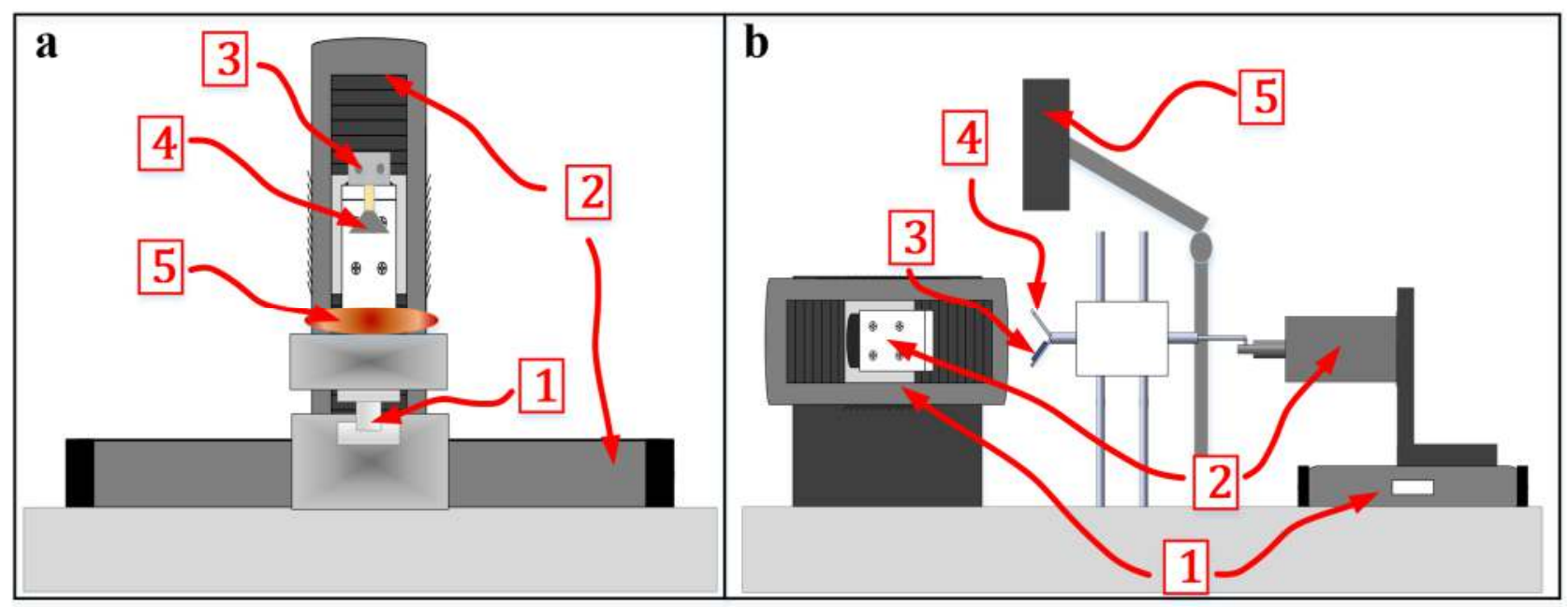

Figure 2. Experimental setups (a) Friction tribometer: 1. 3D force sensor, 2. Linear motorized stages (horizontal and vertical), 3. Load cell, 4. Slider, and 5. Sample. (b) Laparoscopic grasper friction setup: 1. Linear motorized stages, 2. Load cells, 3. Tactile sensor, 4. Tool-tip, and 5. Laser displacement sensor. 


\subsubsection{Friction tribometer}

Coefficient of friction of the flat tip with tactile sensor is measured by friction tribometer illustrated in Fig. 2 (a). A flat slider made of stainless steel is manufactured to the tribometer. Contact area and material of the slider and the flat tool tip are the same. In this custom-made tribometer, two motorized linear stages are used for the vertical (MFA- CC, Newport Corp.) and the horizontal (UTS50CC, Newport Corp.) motion of the slider. A load cell (GSO-250g, Transducer Techniques) and a piezoelectric three-dimensional (3D) force sensor (9317C, Kistler) measure the normal force and the friction force, respectively. A sliding mode controller (SMC) is used to control the normal force applied to tissue throughout the horizontal motion of the slider [16]. Organic samples are from chicken meat in rectangular shapes with a thickness 5 to $8 \mathrm{~mm}$. In order to avoid the contact loss of the sample from the test bench, sandpaper is used. Coefficient of friction are measured under the normal force of $500 \mathrm{mN}, 1000 \mathrm{mN}$, and $2000 \mathrm{mN}$ while velocity of the slider changes between $0.1 \mathrm{~mm} / \mathrm{s}$ and $2.5 \mathrm{~mm} / \mathrm{s}$. Coefficient of friction is calculated from the friction force and SMC-controlled normal force. Total horizontal displacement of the slider for each experiment is $17 \mathrm{~mm}$. For each normal load and sliding velocity, tests are repeated 3 times.

\subsubsection{Laparoscopic grasper friction setup}

In order to measure the coefficient of friction between the tool tip and sample, the laparoscopic grasper friction setup is established. This setup consists of two load cell, a piezoresistive tactile sensor, a laser displacement sensor and two linear motorized stages as illustrated in Fig. 2 (b). The load cell (S2M, HBM) at the right hand side of the Fig. 2 (b), which measures the rod force, is connected to the rod of the tool and to the linear motorized stage, which opens and closes the tool tip. The load cell (GSO-250g, Transducer Techniques) at the left hand side of the Fig. 2 (b), which measures pull force, is connected to the sample with a thread and to the other linear motorized stage, which pulls the sample. Tactile sensor, which measures the pinch force, is integrated to the tool tip. The laser displacement sensor, which is located on top of the tool tip, measures the tip angle $\theta$ as illustrated in Fig.1. The tip angle is kept constant between $1^{\circ}$ and $4^{\circ}$. Details of the pinch force measurement with a tactile sensor are explained in previous studies [17]. The tool is stationary to experiment table. Experiment starts with grasping the sample by sustaining the constant rod force with a proportional $(\mathrm{P})$ controller. Afterwards, the sample is pulled with velocity of $0.5 \mathrm{~mm} / \mathrm{s}$ and it slides between jaws. The rod forces are $3 \mathrm{~N}, 5 \mathrm{~N}$ and $7 \mathrm{~N}$ and the resulting pinch forces are $0.75 \mathrm{~N}, 1 \mathrm{~N}$ and $2 \mathrm{~N}$, respectively. For each velocity and rod force, tests are carried out three times. Coefficient of friction is calculated from the pull force and pinch force. The results of the experiments will be discussed in the following sections.

\subsection{Test Samples}

In order to prevent tissue dehydration, all samples are kept in plastic bags at room temperature $\left(22{ }^{\circ} \mathrm{C}\right)$, and each sample is used only once. Thickness of the samples are between 4 and $6 \mathrm{~mm}$. Test laboratory has constant temperature of $22{ }^{\circ} \mathrm{C}\left( \pm 1^{\circ} \mathrm{C}\right)$ and relative humidity of $65 \%( \pm 3 \%)$. The amount of sample dehydration according to elapsed time is measured with a resistive humidity meter. For the samples, the humidity level of 300 to 700 is suitable for experiments. Once the samples are taken out from the plastic bag, the measurements show that the average humidity of 
the samples is $670 \pm 20$ and the average humidity of the samples decreases roughly $11 \%$ within 15 minutes $(595+5)$. Moreover, average duration of each experiment is 4 minutes. Thus, the effect of dehydration is practically negligible.

\section{Results}

\subsection{Coefficient of Friction in Tribometer}

Coefficient of friction between tool tip and tissue is calculated according to Amontons' law of friction given in Eqn. 1, where $\mathrm{F}, \mu$, and $\mathrm{N}$ are the friction force, coefficient of friction, and normal force, respectively.

$$
F=\mu N
$$

In tribometer, experiments are performed with the stainless steel slider with tactile sensor. The normal force is between $500 \mathrm{mN}$ and $2000 \mathrm{mN}$ and the sliding velocity is between $0.1 \mathrm{~mm} / \mathrm{s}$ and $2.5 \mathrm{~mm} / \mathrm{s}$. Mean and standard deviation of coefficient of friction is calculated after 3 repeated experiments. As illustrated in Fig. 5, coefficient of friction is calculated with respect to displacement for a $500 \mathrm{mN}$ normal force and $2.0 \mathrm{~mm} / \mathrm{s}$ sliding velocity. In order to calculate the average friction of coefficient, nine equally weighted points are selected for each case. Coefficient of friction calculated from Fig. 5 is equal to $2 \mathrm{~mm} / \mathrm{s}$ in Fig. 6 (a). Fig. 6 (a) demonstrates the average and standard deviation of the coefficient of friction under a normal force of $500 \mathrm{mN}$ at sliding velocities of $0.1 \mathrm{~mm} / \mathrm{s}, 0.5 \mathrm{~mm} / \mathrm{s}, 1.0 \mathrm{~mm} / \mathrm{s}, 1.5 \mathrm{~mm} / \mathrm{s}, 2.0 \mathrm{~mm} / \mathrm{s}$, and $2.5 \mathrm{~mm} / \mathrm{s}$. With the same sliding velocities, Fig. 6 (b) and (c) demonstrates the average and standard deviation for the normal force of $1000 \mathrm{mN}$ and $2000 \mathrm{mN}$, respectively.

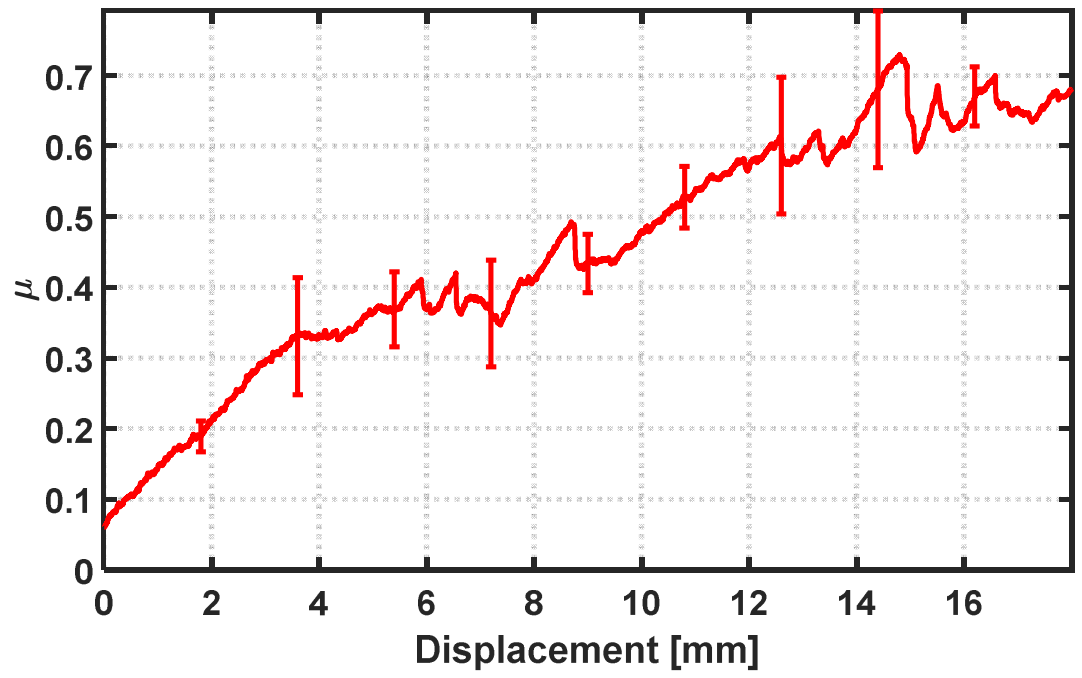

Figure 5. Typical test result in friction tribometer - Coefficient of friction with respect to displacement at $500 \mathrm{mN}$ and $1.0 \mathrm{~mm} / \mathrm{s}$. 
a

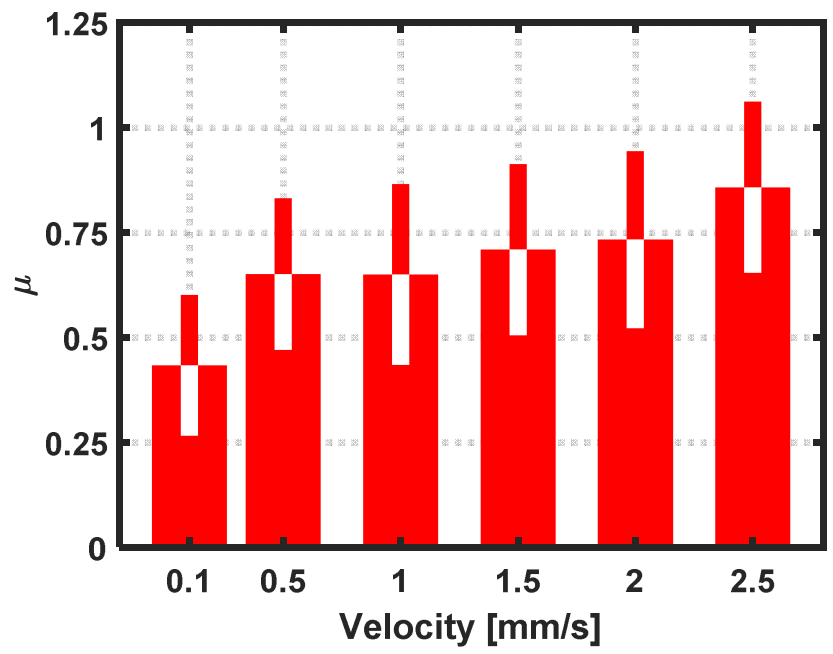

b

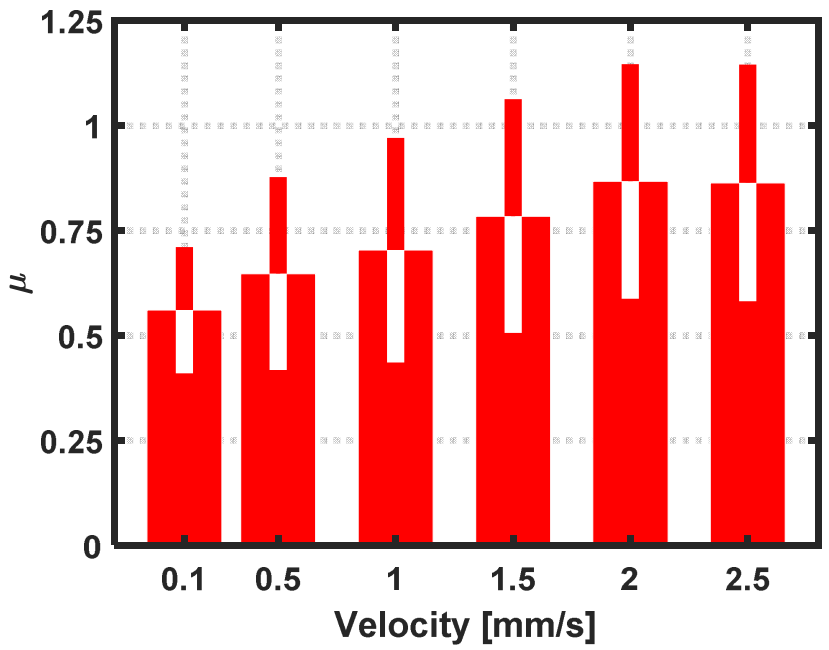

C

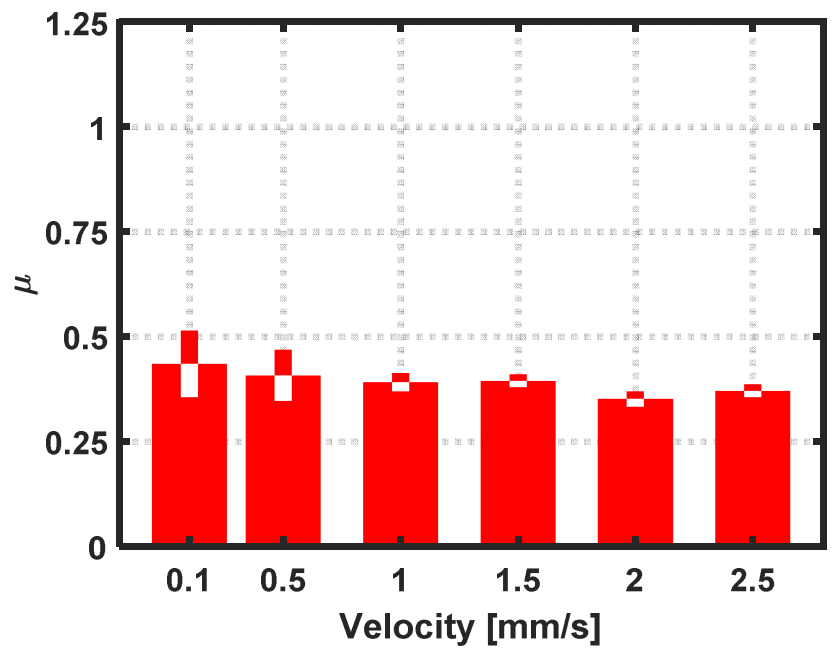

Figure 6. Results of friction tribometer - The coefficient of friction for changing velocities between $0.1 \mathrm{~mm} / \mathrm{s}$ and $2.5 \mathrm{~mm} / \mathrm{s}$ at three different normal force, (a) $500 \mathrm{mN}$, (b) $1000 \mathrm{mN}$, (c) $2000 \mathrm{mN}$. 
As shown in Fig.6 (a) and (b), increasing velocity rises the coefficient of friction at the normal force of $500 \mathrm{mN}$ and $1000 \mathrm{mN}$, respectively. For the $500 \mathrm{mN}$ normal force (Fig.6 (a)), the coefficient of friction rises from 0.43 to 0.85 , as the velocity increases from $0.1 \mathrm{~mm} / \mathrm{s}$ to $2.5 \mathrm{~mm} / \mathrm{s}$. In the same way, for the $1000 \mathrm{mN}$ normal force (Fig. 6 (b)), the velocity increase is from $0.1 \mathrm{~mm} / \mathrm{s}$ to $2.5 \mathrm{~mm} / \mathrm{s}$ and the coefficient of friction rises from 0.56 to 0.86 due to the viscous behavior of the samples. Considering the normal force of $500 \mathrm{mN}$ (Fig.6 (a)) and $1000 \mathrm{mN}$ (Fig.6 (b)), the coefficient of friction increases when the normal force is increased. Moreover, as the velocity is increased from $0.1 \mathrm{~mm} / \mathrm{s}$ to $2.5 \mathrm{~mm} / \mathrm{s}$ at the normal force of $2000 \mathrm{mN}$ (Fig.6 (c)), the coefficient of friction drops from 0.44 to 0.37 . The viscous characteristic of the sample is lost due to disproportionate compression of the sample. In Fig. 6 (c) at $2.5 \mathrm{~mm} / \mathrm{s}$ sliding velocity, coefficient of friction is the lowest. Lower coefficient of friction, which is caused by too much normal force as seen in Fig. 6, rises the possibility of slippage.

\subsection{Coefficient of Friction in Laparoscopic Grasper}

The coefficient of friction amidst the tool-tip and sample are carried out for three different pinch forces in laparoscopic grasper friction setup. The pinch force is maintained constantly at $0.75 \mathrm{~N}$, $1.0 \mathrm{~N}$, and $2.0 \mathrm{~N}$ throughout the test then the sample is pulled. The characteristic test results of the experimental setup is given in Fig. 7. The pull force causes elastic deformation on the sample first then it reaches its highest constant value and it decreases. The coefficient of friction is calculated when the highest constant value of the pull force is achieved. During the experiment, the jaw angle is between $1^{\circ}$ and $4^{\circ}$. Due to latter, pull force is equal to friction force and the pinch force is equal to normal force. The ratio of the pull force to pinch force defined in Eqn.1 is used to calculate the coefficient of friction between the tool-tip and sample. The measurement of the pinch force is not possible without a tactile sensor.

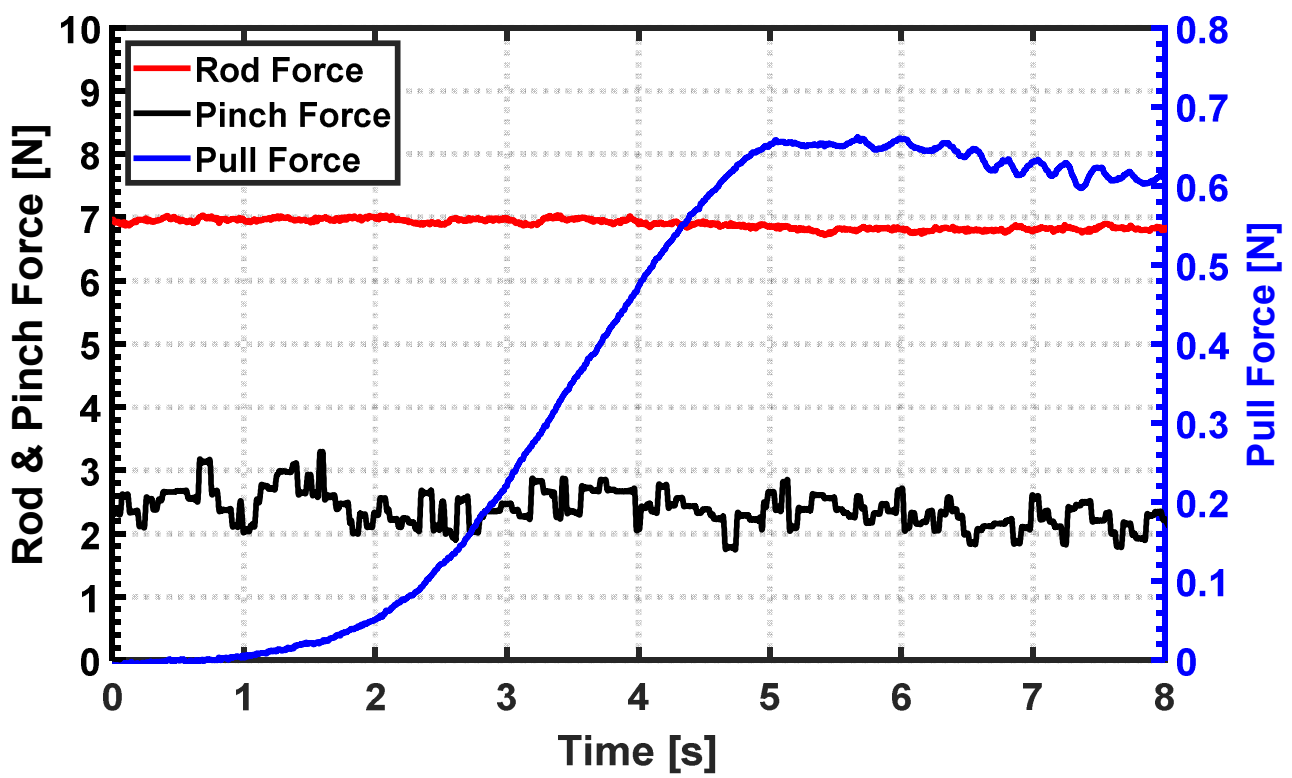

Figure 7. Typical test result of the laparoscopic grasper friction setup - rod, pinch and pull force vs. time.

The results of the experiments are given in Fig. 8 for the sliding velocity of $0.5 \mathrm{~mm} / \mathrm{s}$. The calculated coefficient of friction varies between 0.40 and 0.45 . 


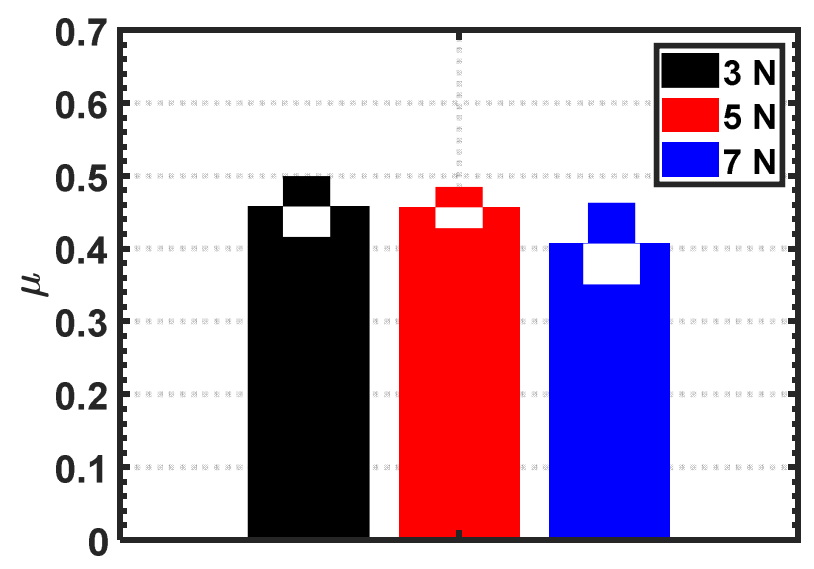

Figure 8. Coefficient of friction calculated by using a tactile sensor.

\section{Discussion}

The coefficient of friction is $80 \%$ higher in tribometer by comparing the results in Fig. 6 for tribometer and in Fig. 8 for laparoscopic grasper setup. During experimental procedures, fresh samples are disposed after each test and variation of their humidity level alternates around $6 \%$. Coefficient of friction of the dry and moderately humid samples are higher than humid samples, which lubricates the tool tip-sample boundary [18-20]. The drying of the sample is avoided by replacing it in each experiment. Additionally, slider shape and contact geometry influences the frictional resistance of the slider thus the coefficient of friction [21]. Comparing the experimental setups, the tip shapes are different which can affect the measured coefficient of frictions. Subsequently, in grasper friction setup, tool tip closes angularly to grasp the sample. Also the squeezed sample sandwiched between tool tip slides when the sample is pulled. However, in the tribometer, the sample is constantly pushed and squeezed along the sliding motion. It could be concluded that the sliding motion of the tips in two different experimental procedure is different and the variation in the coefficient of friction is owing to the different sliding motion. The coefficient of friction obtained in the laparoscopic grasper friction setup is more trustworthy.

\section{Conclusions}

In this study, a sample case is created to evaluate the experimental setups with an organic sample and measure the coefficient of friction of the flat-tipped bowel grasper. Estimation of friction force is important to ensure safe grasping in minimally invasive surgery. The friction tribometer and the laparoscopic grasper setup were two experimental setups to conclude the friction of the tool in the presence of the tactile sensor. The difference in the coefficient of friction between the experimental setups is due to the different sliding motion and slider shape. Comparing the results of the tribometer and the laparoscopic grasper setup, the coefficient of friction is $80 \%$ higher. However, the coefficient of friction obtained in the laparoscopic grasper friction setup is more trustworthy. The measurement of the pinch force and estimation of the friction force from the measured pinch force and the coefficient of friction is suitable for novice surgeon training. 


\section{References}

[1] Hardon SF, Horeman T, Bonjer HJ,. Meijerink WJHJ, Force-based learning curve tracking in fundamental laparoscopic skills training. Surgical Endoscopy. 2018;32(8):3609-3621. Doi:10.1007/s00464-018-6090-7.

[2] Takayasu K, Yoshida K, Kinoshita H, Yoshimoto S, Oshiro O, Matsuda T, Analysis of the tractive force pattern on a knot by force measurement during laparoscopic knot tying. The American Journal of Surgery. Doi:10.1016/j.amjsurg.2017.07.009.

[3] Horeman T, Rodrigues SP, Jansen FW, Dankelman J, van den Dobbelsteen JJ, Force measurement platform for training and assessment of laparoscopic skills. Surgical Endoscopy. 2010;24(12):3102-3108. Doi:10.1007/s00464-010-1096-9.

[4] Cartmill JA, Shakeshaft AJ, Walsh WR, Martin CJ, High Pressures Are Generated At the Tip of Laparoscopic Graspers. ANZ Journal of Surgery. 1999;69(2):127-130.

Doi:10.1046/j.1440-1622.1999.01496.x.

[5] Tiwana MI, Redmond SJ, Lovell NH, A review of tactile sensing technologies with applications in biomedical engineering. Sensors and Actuators, A: Physical. 2012;179:17-31.

Doi:10.1016/j.sna.2012.02.051.

[6] Hassanbeiglou A, Kalantari M, Mozaffari E, Dargahi J, Kövecses J, A new tactile array sensor for viscoelastic tissues with time-dependent behavior. Sensor Review. 2015;35(4):374-381. Doi:10.1108/SR-06-2014-656.

[7] Kalantari M, Ramezanifard M, Ahmadi R, Dargahi J, Kövecses J, A piezoresistive tactile sensor for tissue characterization during catheter-based cardiac surgery. The International Journal of Medical Robotics and Computer Assisted Surgery. 2011;7(4):431-440. Doi:10.1002/rcs.413.

[8] Sümer B, Özin MC, Eray T, Koc IM, The undamaged tissue grasping in a laparoscopic surgical grasper via distributed pressure measurement. Tribology International. 2017;113:330-337. Doi:10.1016/j.triboint.2017.02.033.

[9] Westebring-van der Putten EP, Van den Dobbelsteen JJ, Goossens RHM, Jakimowicz JJ, Dankelman J, Force feedback requirements for efficient laparoscopic grasp control. Ergonomics. 2009;52(9):1055-1066. Doi:10.1080/00140130902912803.

[10] Ayyildiz M, Cinoglu S, Basdogan C, Effect of normal compression on the shear modulus of soft tissue in rheological measurements. Journal of the Mechanical Behavior of Biomedical Materials. 2015;49:235-243. Doi:10.1016/j.jmbbm.2015.05.011. 
[11] Moreira P, Zemiti N, Liu C, Poignet P, Viscoelastic model based force control for soft tissue interaction and its application in physiological motion compensation. Computer Methods and Programs in Biomedicine. 2014;116(2):52-67. Doi:10.1016/j.cmpb.2014.01.017.

[12] Misra S, Ramesh KT, Okamura AM, Modeling of Tool-Tissue Interactions for ComputerBased Surgical Simulation: A Literature Review. Presence (Cambridge, Mass.). 2008;17(5):463. Doi:10.1162/pres.17.5.463.

[13] Castel D, Measurements of the Static Friction Coefficient Between Bone and Muscle Tissues. Journal of Biomechanical Engineering. 2010;132(8). Doi:10.1115/1.4001893.

[14] Kim JS, Sung IH, Kim YT, Kwon EY, Kim DE, Jang YH, Experimental investigation of frictional and viscoelastic properties of intestine for microendoscope application. Tribology Letters. 2006;22(2):143-149. Doi:10.1007/s11249-006-9073-0.

[15] Ramezanifard M, Dargahi J, Najarian S, Narayanan N, Design, Modeling and Fabrication of a Tactile Sensor and Display System for Application in Laparoscopic Surgery. Engineering and Technology. 2009;3(6):209-213.

[16] Sümer B., Influence of preload control on friction force measurement of fabric samples. Tribology International. 2018;127:446-456. Doi:10.1016/j.triboint.2018.06.007.

[17] Koç IM, Eray T, Sümer B, Çerçi N, An active force controlled laparoscopic grasper by using a smart material actuation. Tribology International. 2015;100:317-327. Doi:10.1016/j.triboint.2016.03.001.

[18] Jin Z, Dowson D, Bio-friction, Friction. 2013;1(2):100-113. Doi:10.1007/s40544-013-00044.

[19] Messaoud WB, Bueno MA, Lemaire-Semail B, Relation between human perceived friction and finger friction characteristics. Tribology International. 2016;98:261-269. Doi:10.1016/j.triboint.2016.02.031.

[20] Gueorguiev D, Vezzoli E, Mouraux A, Lemaire-Semail B, Thonnard JL, The tactile perception of transient changes in friction. Journal of The Royal Society Interface. 2017;14 (137) Doi:10.1098/rsif.2017.0641.

[21] Maegawa S, Itoigawa F, Nakamura T, A role of friction-induced torque in sliding friction of rubber materials. Tribology International. 2016; 93:182-189.

Doi:10.1016/j.triboint.2015.08.030. 